\title{
A Polynomial Matrix Inequality Approach for Zonotopic Set-Membership Estimation of Multivariable Systems
}

\author{
V.T.H. Le, C. Stoica, D. Dumur, T. Alamo, E.F. Camacho
}

\begin{abstract}
This paper proposes a methodology for guaranteed state estimation of multivariable linear discrete time systems in the presence of bounded disturbances and noises. A zonotopic outer approximation of the state estimation domain is computed based on the minimization of the $P$-radius associated to the zonotope. The proposed method leads to an off-line Polynomial Matrix Inequality (PMI) optimization problem. A sub-optimal solution of this problem is obtained using relaxation techniques. An illustrative example is analyzed in order to highlight the performance of the proposed algorithm.
\end{abstract}

\section{INTRODUCTION}

State estimation plays an important role in control community. For a nominal system this problem can be easily solved using for example the Luenberger observer. But in general mathematical models are never perfect representations of real systems and the output measure is influenced by noises. In this context, the problem of state estimation becomes more difficult. To solve this problem the Kalman filter [1], which is based on some probabilistic assumptions on perturbations and noises, is widely used. The state is estimated by minimizing the error variance. Despite its conceptual simplicity, these assumptions are sometimes difficult to validate.

The set-membership estimation method (worst-case estimation) can be an alternative. The uncertainties and noises are supposed to be unknown but bounded by some compact sets. The state estimation set is determined containing all the possible system states that are consistent with the uncertain system and the measurement noises. Several geometrical forms can be used to present this state estimation such as ellipsoids [2], [3], [4], [5], [6], polytopes [7] (boxes, parallelotopes [8], zonotopes [9], [10]). In the linear case polytopes can exactly represent the domain of the system state but the complexity grows up exponentially. Therefore a solution is obtained only for polytopes with a reasonable number of vertices. To reduce the complexity, the representation by ellipsoids can be used, but with a loss of performance. In this paper zonotopes (symmetric polytopes) are used to offer a good trade-off between the computational complexity and the precision of the estimation. In addition the wrapping effect (the growth of the domain representation due to uncertainty at each sample time) can be efficiently controlled using

V.T.H. Le, C. Stoica and D. Dumur are with SUPELEC Systems Sciences (E3S) - Automatic Control Department, 3 rue Joliot Curie, F-91192, Gif-sur-Yvette cedex, France (e-mail: \{vutuanhieu.le; cristina.stoica; didier.dumur\}esupelec.fr).

T. Alamo and E.F. Camacho are with Department of Ingeniería de Sistemas y Automática, Universidad de Sevilla, Camino de los Descubrimientos, 41092 Sevilla, Spain (e-mail: alamodcartuja.us.es, eduardo@esi.us.es). zonotopes [11]. Zonotopes are used in several applications such as collision detection [12], reachability analysis [13], state estimation [9], [10], [14], [15].

To estimate the state of linear discrete-time Single-Input Single-Output (SISO) systems with bounded disturbances and measurement noise, [15] presents a new optimization criterion that minimizes the $P$-radius associated to the zonotope in order to obtain good performances and reasonable computation load. This method allows to perform an offline optimization problem which is a major advantage for real-time applications. The originality of the present paper is the generalization of the method presented in [15] for the case of Multi-Input Multi-Output (MIMO) systems with bounded disturbances and measurement noises. This leads to a Polynomial Matrix Inequality (PMI) optimization problem that is difficult to solve. A sub-optimal solution is obtained using the relaxation technique proposed by [16].

This paper is organized as follows. Section II presents some useful mathematical notations and properties. Section III details the proposed solution. An example is analyzed in Section IV to show the effectiveness of the proposed state estimation. Finally, in Section V some concluding remarks and perspectives are drawn.

\section{MATHEMATICAL NotATIONS AND BASIC DEFINITIONS}

This section provides the main notations and definitions and zonotopes' properties necessary for the comprehension of this paper.

An interval $X=[a, b]$ is defined as the set $\{x: a \leq x \leq b\}$. The notations $\operatorname{mid}(X)=\frac{b+a}{2}$ and $\operatorname{rad}(X)=\frac{b-a}{2}$ are used for the center and the radius of the interval $X$. The unitary interval is $\mathbf{B}=[-1,1]$. The set of real compact interval $[a, b]$, where $a, b \in \mathbb{R}$ and $a \leq b$ is denoted $\mathbb{I}$.

A box $\left(\left[a_{1}, b_{1}\right], \ldots,\left[a_{n}, b_{n}\right]\right)^{T}$ is an interval vector. A unitary box in $\mathbb{R}^{m}$, denoted $\mathbf{B}^{m}$, is a box composed by $m$ unitary intervals.

The Minkowski sum of two sets $X$ and $Y$ is defined by $X \oplus Y=\{x+y: x \in X, y \in Y\}$.

Zonotopes are a special class of convex polytopes. A zonotope of order $m$ in $\mathbb{R}^{n}$ can be defined as the linear image $\mathbb{R}^{n}$ of a $m$-dimensional hypercube in $\mathbb{R}^{n}$. The order $m$ is a measure of the geometrical complexity of the zonotopes. Given a vector $p \in \mathbb{R}^{n}$ and a matrix $H \in \mathbb{R}^{n \times m}$ a $m$-zonotope is the set $p \oplus H \mathbf{B}^{m}=\left\{p+H z, z \in \mathbf{B}^{m}\right\}$. This is the Minkowski sum of the $m$-segments defined by 
$m$ columns of matrix $H$ in $\mathbb{R}^{n}$. A centered zonotope is a zonotope whose center is the origin.

The $P$-radius of a zonotope $X=p \oplus H \mathbf{B}^{m}$ is defined as $d(x)=\max \left(\|x-p\|_{P}^{2}\right), x \in X$. This notion is related to the ellipsoid $(x-p)^{T} P(x-p) \leq 1$.

Figure 1 shows a zonotope constructed by a linear image of a centered cube $\left(p=\left[\begin{array}{l}0 \\ 0\end{array}\right]\right.$ ) in $\mathbb{R}^{2}$, with $H=\left[\begin{array}{lll}1 & 2 & 3 \\ 3 & 2 & 1\end{array}\right]$, and the associated P-radius $d(x)=\max \left(\|x\|_{P}^{2}\right)$, with $x \in$ $X=p \oplus H \mathbf{B}^{3}$ and $P=\left[\begin{array}{ll}1 & 0 \\ 0 & 1\end{array}\right]$.

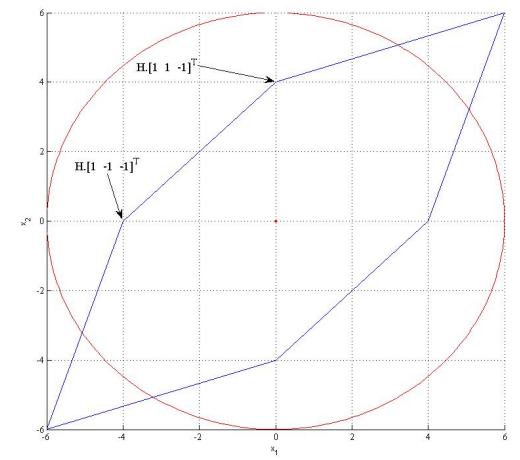

Fig. 1. Zonotope and ellipsoid representing the associated $P$-radius

A strip $X$ is defined as the set $\left\{x \in \mathbb{R}^{n}:\left|c^{T} x-d\right| \leq \sigma\right\}$ with $c \in \mathbb{R}^{n}$ and $d, \sigma \in \mathbb{R}$.

A matrix $M=M^{T} \in \mathbb{R}^{n \times n}$ is called a positivesemidefinite matrix (resp. negative-semidefinite matrix), denoted $M \succeq 0$ (resp. $M \preceq 0$ ), if $z^{T} M z \geq 0$ (resp. $z^{T} M z \leq$ 0 ) for all non-zero vectors $z$ with real entries $\left(z \in \mathbb{R}^{n} \backslash\left\{0_{n}\right\}\right)$.

Property 1: Given two centered zonotopes $Z_{1}=H_{1} \mathbf{B}^{m_{1}} \in \mathbb{R}^{n}$ and $Z_{2}=H_{2} \mathbf{B}^{m_{2}} \in \mathbb{R}^{n}$, the Minkowski sum of two zonotopes is also a zonotope defined by $Z=Z_{1} \oplus Z_{2}=\left[\begin{array}{ll}H_{1} & H_{2}\end{array}\right] \mathbf{B}^{m_{1}+m_{2}}$.

Property 2: The image of a centered zonotope $Z_{1}=H_{1} \mathbf{B}^{m_{1}} \in \mathbb{R}^{n}$ by a linear application $K$ can be computed by a standard matrix product $K \cdot Z_{1}=\left(K \cdot H_{1}\right) \mathbf{B}^{m_{1}}$.

Property 3: (Zonotope reduction) [9], [10] Given the zonotope $Z=p \oplus H \mathbf{B}^{m} \in \mathbb{R}^{n}$ and the integer $s$ with $n<s<m$, denote $\hat{H}$ the matrix resulting from the reordering of the columns of the matrix $H=\left[h_{1} \ldots h_{i} \ldots h_{m}\right]$ in decreasing order of Euclidean norm $\left(\hat{H}=\left[\hat{h}_{1} \ldots \hat{h}_{i} \ldots \hat{h}_{m}\right]\right.$ with $\left.\left\|\hat{h}_{i}\right\|_{2} \geq\left\|\hat{h}_{i+1}\right\|_{2}\right)$. Then $Z \subseteq p \oplus\left[\begin{array}{ll}\hat{H}_{T} & Q\end{array}\right] \mathbf{B}^{s}$, where $\hat{H}_{T}$ is obtained from the first $s-n$ columns of matrix $\hat{H}$ and $Q \in \mathbb{R}^{n \times n}$ is a diagonal matrix satisfying $Q_{i i}=\sum_{j=s-n+1}^{m}\left|\hat{H}_{i j}\right|$, with $i=1, \ldots, n$.

Property 4: ([10]). Given the zonotope $Z=p \oplus H \mathbf{B}^{r}$ $\subset \mathbb{R}^{n_{x}}$, the strip $S=\left\{x \in \mathbb{R}^{n}:\left|c^{T} x-d\right| \leq \sigma\right\}$ and the vector $\lambda \in \mathbb{R}^{n_{x}}$, define a vector $\hat{p}(\lambda)=p+\lambda\left(d-c^{T} p\right) \in \mathbb{R}^{n_{x}}$ and a matrix $\hat{H}(\lambda)=\left[\left(I-\lambda c^{T}\right) H \quad \sigma \lambda\right]$. Then a family of zonotopes (parameterized by the vector $\lambda$ ) that contains the intersection of a zonotope and a strip is obtained such as $X \cap S \subseteq \hat{X}(\lambda)=\hat{p}(\lambda) \oplus \hat{H}(\lambda) B^{r+1}$.

\section{GUARANTEED STATE ESTIMATION USING ZONOTOPES}

This section focuses on the main result of our paper. In a first stage the estimation problem for multivariable discretetime systems is formulated. In a second stage, a solution of this problem is proposed in the form of a Polynomial Matrix Inequality optimization problem. As PMI problems are non convex and hence difficult to solve, a sub-optimal solution is found using the relaxation strategy proposed in [16].

\section{A. Problem Formulation}

Consider the following linear multivariable discrete-time invariant system of the form:

$$
\left\{\begin{array}{l}
x_{k+1}=A x_{k}+\omega_{k} \\
y_{k}=C x_{k}+v_{k}
\end{array}\right.
$$

where $x_{k} \in \mathbb{R}^{n_{x}}$ is the state of the system, $y_{k} \in \mathbb{R}^{n_{y}}$ is the measured output at sample time $k$. The vector $\omega_{k} \in \mathbb{R}^{n_{\omega}}$ represents the state perturbation vector and $v_{k} \in \mathbb{R}^{n_{y}}$ is the measurement perturbation (noise, offset, etc.). It is assumed that the uncertainties and the initial state are bounded by zonotopes: $\omega_{k} \in W, v_{k} \in V$ and $x_{0} \in X_{0}$. To simplify the computation $V$ is assumed to be a box in $\mathbb{R}^{n_{y}}$, the centers of $W$ and $V$ are assumed to be the origin. Note that if this assumption is not satisfied, a change of coordinates can be used. From the definition of zonotopes, $W$ and $V$ can be written as $W=F \mathbf{B}^{n_{\omega}}$ and $V=\Sigma \mathbf{B}^{n_{y}}$, where $F \in \mathbb{R}^{n_{x} \times n_{\omega}}$ and $\Sigma \in \mathbb{R}^{n_{y} \times n_{y}}$ are diagonal matrices.

With these notations, the consistent state set and the exact uncertain set [10] are defined as follows.

Definition 1: Given the system (1) and a measured output $y_{k}$, the consistent state set at time $k$ is defined as $X_{y_{k}}=$ $\left\{x \in \mathbb{R}^{n}:\left(y_{k}-C x\right) \in V\right\}$.

Definition 2: Consider the system (1). The exact uncertain state set $X_{k}=\left(A X_{k-1} \oplus W\right) \cap X_{y_{k}}, k \geq 1$ is equal to the set of states that are consistent with the measured output and the initial state set $X_{0}$.

Remark 1: The exact computation of the uncertain state set is difficult. In practice, this set is approximated by conservative outer bounds to reduce the complexity. An outer approximation using a zonotope-based procedure for SISO systems based on the $P$-radius minimization is presented in [15]. Based on this idea which provides good performance and low computational complexity, an original extended version for MIMO systems will be presented in the following. Let us consider that an outer bound of the exact uncertain state set denoted $\hat{X}_{k-1}$ is available at time instant $k-1$. Suppose also that a measured output $y_{k}$ is obtained at time instant $k$. Under these assumptions, an outer bound of the exact uncertain state set can be estimated with Algorithm 1.

\section{Algorithm 1}

1) Prediction step: Given the system (1), compute a zonotope $\bar{X}_{k}=A \hat{X}_{k-1} \oplus W$ that offers a bound for the uncertain trajectory of the system.

2) Measurement: Compute the consistent state set $X_{y_{k}}$ using the measurements $y_{k}$. 
3) Correction step: To find the uncertain state set, compute an outer approximation $\hat{X}_{k}$ of the intersection between $X_{y_{k}}$ and $\bar{X}_{k}$.

The proposed algorithm is similar to the Kalman filter. To obtain a zonotope bounding the uncertain trajectory of the system in step 1, Properties 1 and 2 are used. The complexity of this zonotope is limited using Property 3 . The intersection set of step 3 is computed via an optimization problem involving the $P$-radius as detailed in the next part.

\section{B. Proposed Solution}

Supposing an outer approximation of the state set $\hat{X}_{k-1}=$ $\hat{p}_{k-1} \oplus \hat{H}_{k-1} \mathbf{B}^{r}$ at the time instant $k-1$, then the predicted state set at the next instant $\bar{X}_{k}$ can be computed using (1), Property 1 and Property 2:

$$
\bar{X}_{k}=A \hat{p}_{k-1} \oplus\left[\begin{array}{ll}
A \hat{H}_{k-1} & F
\end{array}\right] \mathbf{B}^{r+n_{\omega}}=\bar{p}_{k} \oplus \bar{H}_{k} \mathbf{B}^{r+n_{\omega}}
$$

The exact uncertain state set will be obtained after intersecting the predicted state set with the consistent state set. In a general way, the outer approximation of this set can be found by repeating the guaranteed state intersection (Property 4) for each component of the measured output vector $y_{k}$ : $y_{k_{i}}=c_{i}^{T} x_{k}+v_{k_{i}}, i=1, \ldots, n_{y} . c_{i}^{T}$ is the $i$-th row of matrix $C$ and the noise $v_{k_{i}}$ is bounded by the zonotope $V_{i}=\sigma_{i} \mathbb{B}^{1}$, with $\sigma_{i}=\Sigma_{i i} \in \mathbb{R}$.

An outer approximation of the intersection between the consistent state set obtained from the first component of $y_{k}$ and the predicted state set parameterized by a vector $\lambda_{1}$ is represented by:

$$
\hat{X}_{k_{1}}\left(\lambda_{1}\right)=\hat{p}_{k_{1}}\left(\lambda_{1}\right) \oplus \hat{H}_{k_{1}}\left(\lambda_{1}\right) \mathbf{B}^{r+n_{\omega}+1}
$$

where $\hat{p}_{k_{1}}\left(\lambda_{1}\right)=A_{k-1} \hat{p}_{k-1}+\lambda_{1}\left(y_{k_{1}}-c_{1}^{T} A_{k-1} \hat{p}_{k-1}\right)$ and $\hat{H}_{k_{1}}\left(\lambda_{1}\right)=\left[\begin{array}{lll}\left(I-\lambda_{1} c_{1}^{T}\right) A_{k-1} \hat{H}_{k-1} & \left(I-\lambda_{1} c_{1}^{T}\right) F & \sigma_{1} \lambda_{1}\end{array}\right]$. Then this set is intersected with the second component $y_{k_{2}}$ of the measured output vector $y_{k}$ :

$$
\hat{X}_{k_{2}}\left(\lambda_{1}, \lambda_{2}\right)=\hat{p}_{k_{2}}\left(\lambda_{1}, \lambda_{2}\right) \oplus \hat{H}_{k_{2}}\left(\lambda_{1}, \lambda_{2}\right) \mathbf{B}^{r+n_{\omega}+2}
$$

with $\hat{p}_{k_{2}}\left(\lambda_{1}, \lambda_{2}\right)=\hat{p}_{k_{1}}\left(\lambda_{1}\right)+\lambda_{2}\left(y_{k_{2}}-c_{2}^{T} \hat{p}_{k_{1}}\left(\lambda_{1}\right)\right)$ and $\hat{H}_{k_{2}}\left(\lambda_{1}, \lambda_{2}\right)=\left[\left(I-\lambda_{2} c_{2}^{T}\right) \hat{H}_{k_{1}}\left(\lambda_{1}\right) \quad \sigma_{2} \lambda_{2}\right]$. This procedure is repeated until the last component $y_{k_{n_{y}}}$ of the measured output vector $y_{k}$ leading to the zonotopic guaranteed state estimation set at time instant $k$ :

$$
\begin{array}{r}
\hat{X}_{k_{n_{y}}}\left(\lambda_{1}, \ldots, \lambda_{n_{y}}\right)=\hat{p}_{k_{n_{y}}}\left(\lambda_{1}, \ldots, \lambda_{n_{y}}\right) \oplus \\
\oplus \hat{H}_{k_{n_{y}}}\left(\lambda_{1}, \ldots, \lambda_{n_{y}}\right) \mathbf{B}^{r+n_{\omega}+n_{y}}
\end{array}
$$

with

$$
\begin{aligned}
\hat{p}_{k}=\hat{p}_{k_{n_{y}}}( & \left(\lambda_{1}, \ldots, \lambda_{n_{y}}\right)=\hat{p}_{k_{n_{y}-1}}\left(\lambda_{1}, \ldots, \lambda_{n_{y}-1}\right)+ \\
& +\lambda_{n_{y}}\left(y_{k_{n_{y}}}-c_{n_{y}}^{T} \hat{p}_{k_{n_{y}-1}}\left(\lambda_{1}, \ldots, \lambda_{n_{y}-1}\right)\right)
\end{aligned}
$$

and

$$
\begin{aligned}
& \hat{H}_{k}=\hat{H}_{k_{n_{y}}}\left(\lambda_{1}, \ldots, \lambda_{n_{y}}\right)= \\
& \quad=\left[\begin{array}{ll}
\left(I-\lambda_{n_{y}} c_{n_{y}}^{T}\right) \hat{H}_{k_{n_{y}-1}}\left(\lambda_{1}, \ldots, \lambda_{n_{y}-1}\right) & \sigma_{n_{y}} \lambda_{n_{y}}
\end{array}\right]
\end{aligned}
$$

Finally the guaranteed state estimation is represented by a zonotope parameterized by these vectors $\lambda_{1}, \lambda_{2}, \ldots, \lambda_{n_{y}}$.
In order to simultaneously compute the vectors $\lambda_{i}, i=$ $1, \ldots, n_{y}$, the approach considered is the following. Compute a symmetric positive definite matrix $P$ and the vector $\lambda_{i}$ such that at each sample time the $P$-radius of the zonotopic state estimation set is decreased, more precisely the value $\max _{x \in X_{k}}\left(\left\|x-p_{k}\right\|_{P}^{2}\right)$. This means that the zonotopic state estimation set is contracted in time (see Fig. 2) leading to a more precise estimation of the state.

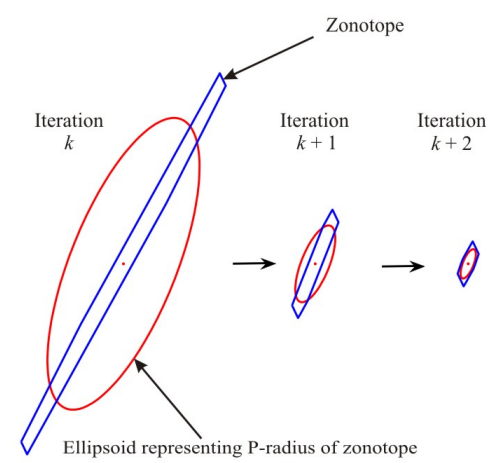

Fig. 2. Evolution of guaranteed state estimation

This condition is described by the following mathematical expression:

$$
\begin{array}{r}
\max _{\hat{z}}\left\|\hat{H}_{k_{n y}} \hat{z}\right\|_{P}^{2} \leq \max _{z} \beta\left\|\hat{H}_{k-1} z\right\|_{P}^{2} \\
+\max _{\omega}\|F \omega\|_{2}^{2}+ \\
+\sigma_{1}^{2}+\ldots+\sigma_{n_{y}}^{2}
\end{array}
$$

with $\hat{z}=\left[\begin{array}{lllll}\tilde{z}^{T} & \tilde{\omega}^{T} & \eta_{1} & \ldots & \eta_{n_{y}}\end{array}\right]^{T} \in \mathbf{B}^{r+n_{\omega}+n_{y}}, \tilde{z}, z \in$ $\mathbf{B}^{r}, \tilde{\omega}, \omega \in \mathbf{B}^{n_{\omega}}, \eta_{i} \in \mathbf{B}^{1}$, and $\beta \in[0,1)$.

Using the explicit form of $\hat{z}$, if the following expression is true, then the expression (8) is also true:

$\max _{\tilde{z}}\left(\left\|\hat{H}_{k_{j}} \hat{z}\right\|_{P}^{2}-\beta\left\|\hat{H}_{k-1} \tilde{z}\right\|_{P}^{2}-\|F \tilde{\omega}\|_{2}^{2}-\sigma_{1}^{2}-\ldots-\sigma_{n_{y}}^{2}\right) \leq 0$

Because $\eta \in \mathbf{B}^{n_{y}}$, the following expression is obtained: $\left[\begin{array}{lll}\sigma_{1}^{2} & \ldots & \sigma_{n_{y}}^{2}\end{array}\right]\left(\left[\begin{array}{c}1 \\ \vdots \\ 1\end{array}\right]-\left[\begin{array}{c}\eta_{1}^{2} \\ \vdots \\ \eta_{n_{y}}^{2}\end{array}\right]\right) \geq 0$. Adding this term to the left of (9) leads to the following sufficient condition for (9):

$$
\begin{array}{r}
\hat{z}^{T} \hat{H}_{k_{j}}^{T} P \hat{H}_{k_{j}} \hat{z}-\beta \tilde{z}^{T} \hat{H}_{k-1}^{T} P \hat{H}_{k-1} \tilde{z}-\tilde{\omega}^{T} F^{T} F \tilde{\omega}- \\
-\sigma_{1}^{2} \eta_{1}^{2}-\ldots-\sigma_{n_{y}}^{2} \eta_{n_{y}}^{2} \leq 0
\end{array}
$$

Denoting $v=\hat{H}_{k-1} \tilde{z}$, then the inequality (10) can be written in the matrix formulation:

$$
\left[\begin{array}{c}
v \\
\tilde{\omega} \\
\eta
\end{array}\right]^{T}\left[\begin{array}{ccccc}
A_{1,1} & A_{1,2} & A_{1,3} & \ldots & A_{1, n_{y}+2} \\
* & A_{2,2} & A_{2,3} & \ldots & A_{2, n_{y}+2} \\
* & * & A_{3,3} & \ldots & A_{3, n_{y}+2} \\
\vdots & \vdots & \vdots & \ddots & \vdots \\
* & * & * & \ldots & A_{n_{y}+2, n_{y}+2}
\end{array}\right]\left[\begin{array}{c}
v \\
\tilde{\omega} \\
\eta
\end{array}\right] \leq 0
$$


with '*' denoting the terms required for the symmetry of the matrix and the following additional notations:

$$
\left\{\begin{array}{l}
A_{1,1}=B_{1} P B_{1}^{T}-\beta P \\
A_{1,2}=B_{1} P B_{2}^{T} \\
A_{1,3}=B_{1} P B_{3}^{T} \\
\vdots \\
A_{1, n_{y}+2}=B_{1} P B_{n_{y}+2}^{T} \\
A_{2,2}=B_{2} P B_{2}^{T}-F^{T} F \\
A_{2,3}=B_{2} P B_{3}^{T} \\
\vdots \\
A_{2, n_{y}+2}=B_{2} P B_{n_{y}+2}^{T} \\
A_{3,3}=B_{3} P B_{3}^{T}-\sigma_{1}^{2} \\
\vdots \\
A_{3, n_{y}+2}=B_{3} P B_{n_{y}+2}^{T} \\
\vdots \\
A_{n_{y}+2, n_{y}+2}=B_{n_{y}+2} P B_{n_{y}+2}^{T}-\sigma_{n_{y}}^{2}
\end{array},\right.
$$

where

$$
\begin{aligned}
& \left.B_{1}=\left(\prod_{i=1}^{n_{y}}\left(I-\lambda_{n_{y}+1-i} c_{n_{y}+1-i}^{T}\right)\right) A\right)^{T} \\
& \left.B_{2}=\left(\prod_{i=1}^{n_{y}}\left(I-\lambda_{n_{y}+1-i} c_{n_{y}+1-i}^{T}\right)\right) F\right)^{T} \\
& B_{3}=\left(\prod_{i=1}^{n_{y}-1}\left(I-\lambda_{n_{y}+1-i} c_{n_{y}+1-i}^{T}\right) \sigma_{1} \lambda_{1}\right)^{T} \\
& \vdots \\
& B_{n_{y}}=\left(\left(I-\lambda_{n_{y}} c_{n_{y}}^{T}\right)\left(I-\lambda_{n_{y}-1} c_{n_{y}-1}^{T}\right) \sigma_{n_{y}-2} \lambda_{n_{y}-2}\right)^{T} \\
& B_{n_{y}+1}=\left(\left(I-\lambda_{n_{y}} c_{n_{y}}^{T}\right) \sigma_{n_{y}-1} \lambda_{n_{y}-1}\right)^{T} \\
& B_{n_{y}+2}=\left(\sigma_{n_{y}} \lambda_{n_{y}}\right)^{T} .
\end{aligned}
$$

Using the definition of positive definite matrix allows to rewrite (11) as:

$$
\left[\begin{array}{cccc}
A_{1,1} & A_{1,2} & \ldots & A_{1, n_{y}+2} \\
* & A_{2,2} & \ldots & A_{2, n_{y}+2} \\
\vdots & \vdots & \ddots & \vdots \\
* & * & \ldots & A_{n_{y}+2, n_{y}+2}
\end{array}\right] \preceq 0, \quad \forall\left[\begin{array}{c}
v \\
\tilde{\omega} \\
\eta
\end{array}\right] \neq 0
$$

or

$$
\left[\begin{array}{cccc}
-A_{1,1} & -A_{1,2} & \ldots & -A_{1, n_{y}+2} \\
* & -A_{2,2} & \ldots & -A_{2, n_{y}+2} \\
\vdots & \vdots & \ddots & \vdots \\
* & * & \ldots & -A_{n_{y}+2, n_{y}+2}
\end{array}\right] \succeq 0, \quad \forall\left[\begin{array}{c}
v \\
\tilde{\omega} \\
\eta
\end{array}\right] \neq 0
$$

Doing some manipulations in (15) leads to:

$$
\begin{gathered}
{\left[\begin{array}{ccccc}
\beta P & 0 & 0 & \ldots & 0 \\
* & F^{T} F & 0 & \ldots & 0 \\
* & * & \sigma_{1}^{2} & \ldots & 0 \\
\vdots & \vdots & \vdots & \ddots & 0 \\
* & * & * & \ldots & \sigma_{n_{y}}^{2}
\end{array}\right]-} \\
-\left[\begin{array}{c}
B_{1} P \\
B_{2} P \\
\vdots \\
B_{n_{y}+2} P
\end{array}\right] P^{-1}\left[\begin{array}{c}
B_{1} P \\
B_{2} P \\
\vdots \\
B_{n_{y}+2} P
\end{array}\right]^{T} \succeq 0
\end{gathered}
$$

Using the Schur complement [17], (16) is equivalent to the following Polynomial Matrix Inequality problem:

$$
\left[\begin{array}{cccccc}
\beta P & 0 & 0 & \ldots & 0 & B_{1} P \\
* & F^{T} F & 0 & \ldots & 0 & B_{2} P \\
* & * & \sigma_{1}^{2} & \ldots & 0 & B_{3} P \\
\vdots & \vdots & \vdots & \ddots & \vdots & \vdots \\
* & * & * & \ldots & \sigma_{n_{y}}^{2} & B_{n_{y}+2} P \\
* & * & * & \ldots & * & P
\end{array}\right] \succeq 0
$$

where the decision variables are $\lambda_{1}, \ldots, \lambda_{n_{y}}, P$ and $\beta$.

Denote the $P$-radius of the state estimation set at instant $k$ as $L_{k}=\max _{x}\left(\left\|x-p_{k}\right\|_{P}^{2}\right)$, where $x \in \hat{X}_{k}$. As the 2norm is a convex function and $W$ is a convex set the term $\max _{\omega}\|F \omega\|_{2}^{2}$ can be easily computed. Denoted const $=$ $\max _{\omega}\|F \omega\|_{2}^{2}+\sigma_{1}^{2}+\ldots+\sigma_{n_{y}}^{2}$ Then the condition (8) can be written as $L_{k+1} \leq \beta L_{k}+$ const. At infinity, this expression is equivalent to:

$$
L_{\infty}=\beta L_{\infty}+\text { const }
$$

leading to

$$
L_{\infty}=\frac{\text { const }}{1-\beta}
$$

Let us consider an ellipsoid $E=\left\{x: x^{T} P x \leq \frac{\text { const }}{1-\beta}\right\}$ which is equivalent to $E=\left\{x: x^{T} \frac{(1-\beta) P}{\text { const }} x \leq 1\right\}$. To minimize the $P$-radius (i.e. $L_{\infty}$ ) of the zonotope, the ellipsoid of smallest diameter must be found ([17]) solving the following optimization problem:

$$
\max _{\tau, P, \beta, \lambda_{1}, \ldots, \lambda_{n_{y}}} \tau
$$

subject to

$$
\left\{\begin{array}{l}
0 \leq \beta<1 \\
\frac{(1-\beta) P}{\text { const }} \succeq \tau I \\
{\left[\begin{array}{cccccc}
\beta P & 0 & 0 & \ldots & 0 & B_{1} P \\
* & F^{T} F & 0 & \ldots & 0 & B_{2} P \\
* & * & \sigma_{1}^{2} & \ldots & 0 & B_{3} P \\
\vdots & \vdots & \vdots & \ddots & \vdots & \vdots \\
* & * & * & \ldots & \sigma_{n_{y}}^{2} & B_{n_{y}+2} P \\
* & * & * & \ldots & * & P
\end{array}\right] \succeq 0}
\end{array}\right.
$$

In this PMI problem, the decision variables are: $P=$ $P^{T} \in \mathbb{R}^{n_{x} \times n_{x}}, \lambda_{1}, \ldots, \lambda_{n_{y}} \in \mathbb{R}^{n_{x}}$ and $\beta, \tau \in \mathbb{R}$. Thus the total number of scalar decision variables is: $\frac{n_{x}\left(n_{x}+1\right)}{2}+n_{y} n_{x}+2$. The degree of the PMI (17) is $n_{y}+1$. 
This PMI can be solved by using the Linear Matrix Inequality (LMI) relaxation methodology [16] that will be detailed in the next part.

\section{Relaxation of the PMI problem}

In a first stage, all of the $\frac{n_{x}\left(n_{x}+1\right)}{2}+n_{y} n_{x}+2$ scalar decision variables are denoted as follows: $\tau, y_{10 \ldots 0}, y_{01 \ldots 0}$, $\ldots, y_{00 \ldots 10}, y_{00 \ldots 01}$. For expression (20), this leads to $\beta=y_{100 \ldots 0}, P=\left[\begin{array}{ccc}y_{01 \ldots 0} & \ldots & y_{00 \ldots 1 \ldots 0} \ldots \\ \ldots & \ldots & \ldots\end{array}\right], \quad \lambda_{1}^{T}=$ $\left[\begin{array}{ll}y_{00} \ldots 1 \ldots 0 & \ldots .\end{array}\right], \ldots, \lambda_{n_{y}}^{T}=\left[\begin{array}{lll}y_{00 \ldots 1 \ldots 0} & \ldots & y_{00} \ldots 01\end{array}\right]$.

In a second stage, the polynomial decision elements in (20) are rewritten as the result of a change of variables based on the previous scalar decision variables such as: $y_{20 \ldots 0}=y_{10 \ldots 0} \cdot y_{10 \ldots 0}, y_{11 \ldots 0}=y_{10 \ldots 0} \cdot y_{01 \ldots 0}$ etc. In this way, expression (20) becomes a LMI. If the LMI relaxation of the PMI optimization problem (20) is used, then the following LMI problem must be solved (see [16] for more details):

$\max \tau$

subject to the LMIs

$$
\left\{\begin{array}{c}
M_{1}=\left[\begin{array}{cccc}
1 & * & * & * \\
y_{10 \ldots 0} & y_{20 \ldots 0} & \ldots & * \\
y_{01 \ldots 0} & y_{11 \ldots 0} & \ldots & * \\
\vdots & \vdots & \ddots & \vdots \\
y_{00 \ldots 111} & \ldots & \ldots & y_{00 \ldots 222}
\end{array}\right] \succeq 0 \\
M_{2} \succeq 0
\end{array}\right.
$$

where $M_{2}$ denotes the equivalent LMI expressions obtained from the PMI in (20) using the new scalar decision variables.

Denote $l=\frac{n_{x}\left(n_{x}+1\right)}{2}+n_{y} n_{x}+1$. As the degree of the PMI in (20) is $n_{y}+1$ thus the dimension of LMIs (21) are:

- $M_{1}: q \times q$, with $q=1+l+\left(\begin{array}{l}2 \\ l\end{array}\right)+\ldots+\left(\begin{array}{r}\operatorname{round}\left(\frac{n_{y}+1}{2}\right) \\ l\end{array}\right)$;

- $M_{2}: 1, n_{x} \times n_{x},\left(2 n_{x}+n_{\omega}+n_{y}\right) \times\left(2 n_{x}+n_{\omega}+n_{y}\right)$.

The number of scalar decision variables in this optimization problem is $\frac{q(q+1)}{2}+1$.

Remark 2: The prediction step using zonotopes is a very simple matrix computation. However, this computation increases the order of the zonotope at each step. In order to control the domain complexity, a reduction step is implemented to bound a high-order zonotope by a lower-order zonotope using Property 3.

Remark 3: The PMI problem (20) can be also solved using an inner approximation [18] instead of the outer approximation [16] proposed in this section.

Remark 4: Some LMI optimizations must be solved to obtain the value of $\lambda_{i}$ but this computation is done off-line.

In order to illustrate the effectiveness of this technique, it will be applied on an example in the next section.

\section{ILLUSTRATIVE EXAMPLE}

Consider the following linear discrete time system:

$$
\left\{\begin{array}{l}
x_{k+1}=\left[\begin{array}{cc}
0 & -0.5 \\
1 & 1
\end{array}\right] x_{k}+0.02\left[\begin{array}{c}
-6 \\
1
\end{array}\right] \omega_{k} \\
y_{k}=\left[\begin{array}{cc}
-2 & 1 \\
1 & 1
\end{array}\right] x_{k}+0.2 v_{k}
\end{array}\right.
$$

with $\left\|v_{k}\right\|_{\infty} \leq 1,\left\|\omega_{k}\right\|_{\infty} \leq 1$. The values of $v_{k}$ and $\omega_{k}$ are generated by random functions. The initial state belongs to the box $3 B^{2}$. The order of the zonotopes is limited to $m \leq 20$ in the interest of a fast simulation. A simple system of $2^{\text {nd }}$ order $\left(n_{x}=2, n_{y}=2\right)$ is chosen in order to reduce the complexity of computation and to facilitate the graphic visualization.

Denote $\beta=y_{10000000}, P=\left[\begin{array}{cc}y_{01000000} & y_{00100000} \\ * & y_{00010000}\end{array}\right]$, $\lambda_{1}^{T}=\left[\begin{array}{ll}y_{00001000} & y_{00000100}\end{array}\right], \lambda_{2}^{T}=\left[\begin{array}{ll}y_{00000010} & y_{00000001}\end{array}\right]$. Thus the dimensions of LMIs of the considered optimization problem (21) are:

- $M_{1}: 37 \times 37$;

- $M_{2}: 1,2 \times 2,7 \times 7$.

As $l=8$ and $q=37$, the total scalar number of decision variables is $\frac{37 \cdot 38}{2}+1=704$.

Figures 3 and 4 show the evolution of the predicted state set and the outer approximation of the state estimation set. This outer approximation is reduced at each iteration. Note that the obtained set is an approximation of the real intersection which will become more and more accurate at each iteration (Fig. 3, Fig. 4).

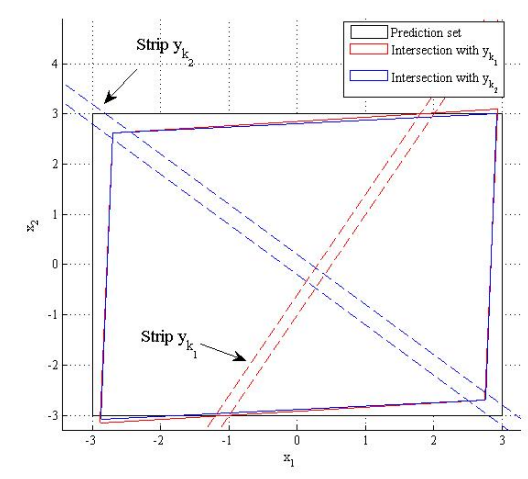

Fig. 3. Intersection $\hat{X}_{k}$ between the predicted state set $\bar{X}_{k}$ and the measurement $X_{y_{k}}$ at the time instant $k=1$

Figures 5 and 6 present the obtained bounds on $x_{1_{k}}$ and $x_{2_{k}}$. The stars represent the real state $x_{1_{k}}, x_{2_{k}}$ of the system. These points are found between the bounds of $x_{1_{k}}, x_{2_{k}}$ which confirms that these bounds are well estimated. Figure 7 shows the evolution of the volume of the guaranteed state estimation. The volume of the zonotopes decreases in time, which confirms the contractive property of the proposed method. 


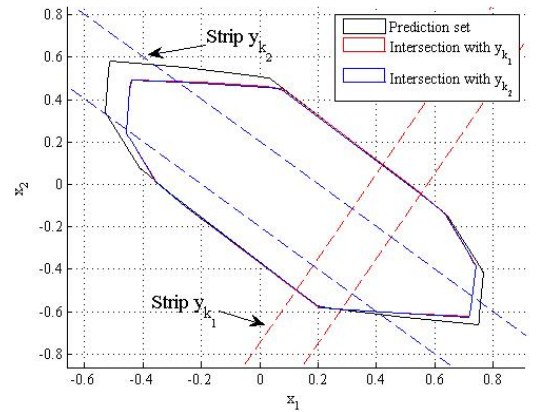

Fig. 4. Intersection $\hat{X}_{k}$ between the predicted state set $\bar{X}_{k}$ and the measurement $X_{y_{k}}$ at the time instant $k=8$

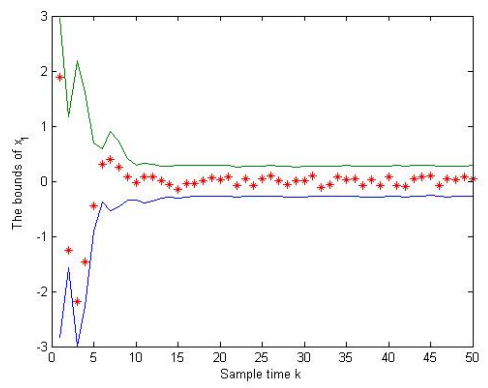

Fig. 5. Bounds of $x_{1}$

\section{CONCLusion}

In this paper a new zonotopic outer-bounding of the state estimation for a multivariable linear discrete time invariant system has been proposed. The proposed method computes a set of all the states that are consistent with the measured output, the bounded noise, the bounded perturbation. The contractive condition on the guaranteed state estimation leads to a Polynomial Matrix Inequality optimization problem. A sub-optimal solution of this PMI problem is obtained using a relaxation technique [16] that leads to a Linear Matrix Inequality optimization problem.

Current work focuses on different strategies for solving the PMI problem in order to improve this result. An interesting perspective is to extend the zonotopic state estimation to the case of multivariable uncertain systems, in the presence of bounded disturbances and noises.

\section{REFERENCES}

[1] R. E. Kalman, "A new approach to linear filtering and prediction problems," Transactions of the ASME-Journal of Basic Engineering, vol. 82, no. Series D, pp. 35-45, 1960.

[2] F. C. Schweppe, "Recursive state estimation: Unknown but bounded errors and system inputs," IEEE Trans. Automat. Contr., vol. 13(1), pp. 22-28, 1968

[3] S. H. Witsenhausen, "Sets of possible states of linear systems given perturbed observations," IEEE Trans. Automat. Contr., vol. 13, pp. 556-558, 1968.

[4] D. P. Bertsekas and I. B. Rhodes, "Recursive state estimation for a setmembership description of uncertainty," IEEE Trans. Automat. Contr., vol. 16(2), pp. 117-128, 1971.

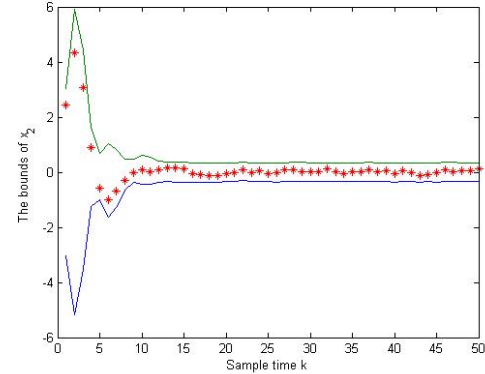

Fig. 6. Bounds of $x_{2}$

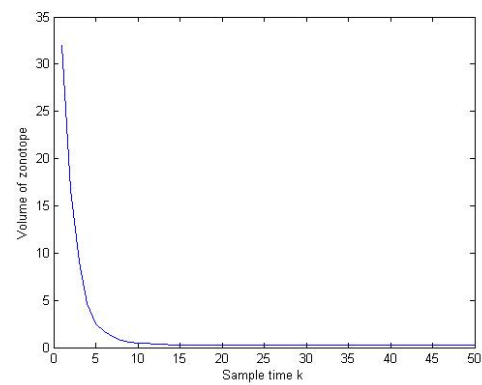

Fig. 7. Evolution of the volume of the guaranteed state estimation

[5] C. Durieu, E. Walter, and B. Polyak, "Multi-input multi-output ellipsoidal state bounding," Journal of optimization theory and applications, vol. 111(2), pp. 273-303, 2001.

[6] B. T. Polyak, S. A. Nazin, C. Durieu, and E. Walter, "Ellipsoidal parameter or state estimation under model uncertainty," Automatica, vol. 40, pp. 1171-1179, 2004.

[7] E. Walter and H. Piet-Lahanier, "Exact recursive polyhedral description of the feasible parameter set for bounded-error models," IEEE Trans. Automat. Contr., vol. 34(8), pp. 911-915, 1989.

[8] A. Vicino and G. Zappa, "Sequential approximation of feasible parameter sets for identification with set membership uncertainty," IEEE Trans. Automat. Contr., vol. 41, pp. 774-785, 1996.

[9] C. Combastel, "A state bounding observer based on zonotopes," in Proc. of European Control Conference, Cambridge, UK, 2003.

[10] T. Alamo, J. M. Bravo, and E. F. Camacho, "Guaranteed state estimation by zonotopes," Automatica, vol. 41, pp. 1035-1043, 2005.

[11] W. Kühn, "Rigorously computed orbits of dynamical systems without the wrapping effect," Computing, vol. 61, pp. 47-67, 1998.

[12] L. J. Guibas, A. Nguyen, and L. Zhang, "Zonotopes as bounding volume," in Proc. of the Symposium on Discrete Algorithm, 2005, pp. 803-812.

[13] M. Althoff, O. Stursberg, and M. Buss, "Reachability analysis of linear systems with uncertain parameters and inputs," in Proc. of the 46th IEEE Conference on Decision and Control, vol. 41, 2007, pp. 726732, New Orleans, LA, USA.

[14] V. Puig, P. Cugueró, and J. Quevedo, "Worst-case estimation and simulation of uncertain discrete-time systems using zonotopes," in Proc. of Europeen Control Conference, Portugal, 2001.

[15] V. Le, T. Alamo, E. Camacho, C. Stoica, and D. Dumur, "A new approach for guaranteed state estimation by zonotopes," in Proc. of the 18th World Congress IFAC, Milan, Italy, 2011.

[16] D. Henrion and J. B. Lasserre, "Convergent relaxations of polynomial matrix inequalities and static output feedback," IEEE Transactions on Automatic Control, vol. 51(2), pp. 192 - 202, 2006.

[17] S. Boyd, L. E. Ghaoui, E. Feron, and V. Balakrishnan, Linear Matrix Inequalities in System and Control Theory. Philadelphia: SIAM, 1994.

[18] D. Henrion and J. B. Lasserre, "Inner approximations for polynomial matrix inequalities and robust stability regions," in LAAS-report 11210 , 2011. 